\title{
Proliferation cell nuclear antigen (clone 19A2) correlates with 5-bromo-2-deoxyuridine labelling in human colonic epithelium
}

\author{
U M Weisgerber, H Boeing, R Nemitz, R Raedsch, R Waldherr
}

\begin{abstract}
Measurements of cell proliferation can be used as biomarkers of preneoplastic change. In this study, two immunocytochemical methods that measure different components of the cell cycle were compared to assess cell proliferation on biopsy samples from human colonic mucosa. These methods are based on a monoclonal antibody against 5-bromo-2-deoxyuridine (BrdU), which is confined to $S$ phase cells, and a more broad assessment of proliferation based on an antibody against proliferating cell nuclear antigen (PCNA, clone 19A2). In the PCNA assay, only strongly immunostained nuclei were included. The proliferation index was assessed in colonic mucosa from patients with no colonic disorders. Correlation between individual total proliferation indices determined by either method was significant with $r_{s}=0.6(p<0.05)$. The mean proliferation index in the study group was $7 \cdot 79 \%$ using BrdU and $7.64 \%$ using PCNA immunocytochemistry. Distribution of labelled cells within crypts was similar with respect to the two methods with a peak at the 18th and the 24th percentile in the case of BrdU and at the 23rd percentile for PCNA. Variance component analysis showed that at least two biopsy specimens should be evaluated per subject to allow a precise individual characterisation. It is concluded that PCNA (19A2) immunocytochemistry may be used as an operational marker of cell proliferation in normal colonic mucosa. A significant correlation and an agreement in the mean proliferation index between PCNA (19A2) and BrdU can only be achieved by a strictly standardised enumeration of labelled cells limited to strongly stained nuclei in the PCNA evaluation.

(Gut 1993; 34: 1587-1592)
\end{abstract}

Cancer Research Center, Heidelberg, Germany

$\mathrm{U} M$ Weisgerber

H Boeing

Institute of Pathology, University of Heidelberg, Heidelberg

R Nemitz

$\mathrm{R}$ Waldherr

Department of

Gastroenterology,

University of Heidelberg,

Heidelberg

R Raedsch

Correspondence to:

Dr U M Weisgerber, German Cancer Research Center, Division of Epidemiology, Im Neuenheimer Feld 280,
D-69120 Heidelberg, Germany.

Accepted for publication 4 March 1993

Biological markers are becoming increasingly important for early detection of an increased cancer risk and as intermediate end points for chemoprevention trials.' Many studies have shown the importance of cell kinetic parameters in the progression of colorectal cancer. ${ }^{2-5}$

Methods that detect the incorporation of a DNA precursor such as $\left[{ }^{3} \mathrm{H}\right]$ - thymidine labelling $^{6}$ or immunocytochemical detection of 5-bromo-2-deoxyuridine $(\mathrm{BrdU})^{7}$ are direct and confine measurements of DNA synthesis in active $S$ phase proliferating cells. They are labour intensive, however, and complex to perform on a large scale, for example, in clinical regimens. Proliferation markers based on the immunocytochemical finding of endogenous antigens such as $\mathrm{Ki}^{\circ} 7^{\circ}$ or proliferating cell nuclear antigen (PCNA) generally express a wider range of the cell cycle, but their application in clinical practice is considerably less complex. The simplicity is particularly true for PCNA, because the antibody can be applied in paraffin embedded tissue.

PCNA is a cell cycle related protein, which is expressed increasingly through $\mathrm{Gl}$ phase, reaching its maximum in early $\mathrm{S}$ phase, and persisting with decreasing detectability in G2 phase. ${ }^{10}$ Bravo and MacDonald-Bravo ${ }^{11}$ described a soluble form of PCNA, which is lost on fixation in organic solvents and does not participate in DNA replication, and an insoluble form, which is associated with the sites of ongoing DNA synthesis and is expressed particularly during $S$ phase and is detectable by immunofluorescence. Bravo and MacDonald-Bravo" report that the soluble form is not detected by immunofluorescence if methanol is used for fixation. Moreover, various PCNA antibodies define different epitopes of PCNA and fixation conditions may affect epitope location within cells. ${ }^{12}$ Therefore, PCNA may serve as a useful marker for proliferation if the conditions of fixation are held constant and the same antibody is used throughout the experiment.

Use of a monoclonal antibody to PCNA in investigative studies requires standardisation of the method in relation to established methods for measuring cell proliferation. Comparative studies have shown that PCNA provides a strong correlation with $\left[{ }^{3} \mathrm{H}\right]$-thymidine labelling and flow cytometric assessment of $S$ phase fraction in rat colon ${ }^{1314}$ and with autoradiographic labelling in human tissue. ${ }^{15} 16$ The determination of proliferation indices in colonic epithelium by $\mathrm{BrdU}$ incorporation in colonic biopsies and subsequent immunocytochemical detection has been found to be equivalent to the standard autoradiographic method by our group (unpublished data).
In this study, we describe use of a monoclonal antibody (19A2) against PCNA on methanol fixed, paraffin embedded human colonic biopsy specimens using a routine biotin streptavidin immunocytochemical system. Measures of proliferative activity were compared with results obtained by BrdU incorporation and subsequent $\mathrm{ABC}$ immunocytochemistry in the same group of patients. We decided to use the term 'proliferation index' to describe the results of the PCNA assay because the antibody to PCNA does not label only S phase cells; we use the same term for the results of the BrdU assay, although the term 'labelling index' is frequently used by other groups. The aim of this study was to evaluate 
how the proliferation index obtained by PCNA (19A2) correlates with that determined by conventional BrdU immunocytochemistry.

\section{Subjects and methods}

SUBJECTS

The study group consisted of 17 subjects (10 women, 7 men) with a mean age of 47 years (range, 19-75). All subjects had a normal diet and were free of inflammatory bowel disease and of benign and malignant tumours at the time of complete colonoscopy. Personal and family anamneses for colorectal cancer (first degree relatives) were negative in all patients. Histological evaluation showed normal mucosa. Bowel preparation was performed by oral administration of two litres of a PEG electrolyte solution. This preparation has been shown not to affect epithelial cell proliferation. ${ }^{17}$ The study was approved by the ethical committee of the University of Heidelberg.

A minimum of four biopsy samples were obtained from each patient from macroscopically normal mucosa at the rectosigmoidal junction (about $20 \mathrm{~cm}$ from the anal verge), using standard forceps. The samples were distributed equally to the BrdU and PCNA protocols.

\section{BRDU PROTOCOL}

The biopsy specimens were immersed immediately in Dulbecco's modified Eagle's medium (Serva, Heidelberg), prewarmed to $37^{\circ} \mathrm{C}$, with the mucosal side upwards and placed on a filter paper under a dissecting microscope. Incubation was conducted in small Petri dishes containing $10 \mathrm{ml}$ of Eagle's medium containing $10 \%$ fetal calf serum and $160 \mu \mathrm{M}$ BrdU (Amersham, Braunschweig, Germany) for two hours at $37^{\circ} \mathrm{C}$. The biopsy specimens were then washed with phosphate buffered saline (PBS) and fixed overnight (at least 18 hours) in absolute methanol at $4^{\circ} \mathrm{C}$. After dehydration and removal of the filter paper, the flat, fixed biopsy specimens were oriented in a vertical position in one paraffin block. Specimens were embedded in paraplast with a melting temperature of $54-56^{\circ} \mathrm{C}$ (Klinipath, Zevenaar, Netherlands).

Between 80 and 100 serial sections $(3 \mu \mathrm{m}$ thick) were cut on a rotary microtome and mounted on glass slides. Because the lumen of the same crypt is visible within a maximal range of $50 \mu \mathrm{m},{ }^{18}$ every 15 th section was stained and counted to ensure that the same crypt was not counted twice. The slides were dried overnight at $37^{\circ} \mathrm{C}$ for better adherence of the sections. Sections were dewaxed in xylene and rehydrated through graded ethanol to $0 \cdot 01 \mathrm{M}$ PBS, $\mathrm{pH} 7 \cdot 4$. Endogenous peroxidase activity was blocked with $0.3 \%$ hydrogen peroxide in $0.1 \%$ sodium azide for $\mathbf{3 0}$ minutes at room temperature. After rinsing in PBS, acid hydrolysis was performed by incubating the sections in $2 \mathrm{~N} \mathrm{HCl}$ for 30 minutes at room temperature followed by washing in two successive baths (three minutes each) of $0.1 \mathrm{M}$ borax $\left(\mathrm{Na}_{2} \mathrm{~B}_{4} \mathrm{O}_{7}\right)$ at $\mathrm{pH} 8.5$ to neutralise the acid.

After being washed in PBS (twice for five minutes), the slides were incubated overnight with mouse monoclonal antibody against BrdU (Amersham, Braunschweig, Germany) at $4^{\circ} \mathrm{C}$ in a humid chamber. The sections were then incubated with biotinylated anti-mouse immunoglobulins from sheep (1:50 for 30 minutes; Amersham), followed by incubation with streptavidin biotinylated peroxidase complex (1:100 for 30 minutes; Amersham). Biotinylated anti-mouse immunoglobulins and streptavidin biotinylated peroxidase complex were diluted in PBS containing 1\% human IgG. After each step, the sections were rinsed in PBS ( $3 \times 5$ minutes).

The reaction product was visualised using a substrate solution containing $0.05 \% \quad 3,3$ diaminobenzidinetetrahydrochloride (DAB) (Serva, Heidelberg, Germany), 0.01\% hydrogen peroxide, $0.01 \%$ nickel chloride, and $0.01 \%$ cobalt chloride in TRIS buffered saline ( $\mathrm{pH} \mathrm{7 \cdot 6)}$ with a usual reaction time of 10 minutes under microscopic examination. After being washed in distilled water, the slides were counterstained with $0 \cdot 1 \%$ nuclear fast red in $5 \%$ aluminium sulphate for five minutes, dehydrated, and mounted using a synthetic mounting medium (Vitro-Clud, Langenbrink, Emmendingen, Germany).

PCNA PROTOCOL

For the PCNA immunoreaction, biopsy specimens were washed in PBS, flattened on a filter paper for better orientation after fixation, and fixed overnight in absolute methanol at $4^{\circ} \mathrm{C}$. Before establishing the final protocol, various tissue fixation methods were compared to investigate their influence on staining intensity for both the PCNA and the BrdU protocol. As an alternative to absolute methanol, biopsy samples were subdivided and fixed for 18-24 hours in $10 \%$ buffered formalin, $70 \%$ ethanol, $70 \%$ methanol, and a commercially available alcoholic fixative called 'Primafix'. Subsequent tissue handling was the same as that in the BrdU protocol.

In the immunocytochemical staining procedure, sections are digested gently by incubation in $0.02 \%$ Saponin for 30 minutes at room temperature (Wargovich, personal communication). This step replaces DNA denaturation by acid hydrolysis with subsequent neutralisation in the BrdU protocol.

After the sections had been washed in PBS, a mouse monoclonal antibody against PCNA (IgM isotype, clone 19A2; Camon, Wiesbaden, Germany) was applied and incubated overnight at $4^{\circ} \mathrm{C}$ in a humid chamber. Biotinylated antimouse immunoglobulins, streptavidin biotinylated peroxidase complex, DAB reaction, and the final counterstaining were conducted as described for the BrdU protocol.

\section{DETERMINATION OF PROLIFERATION INDEX}

All sections were examined by one observer (RN) who had no knowledge of the clinical features or the histological diagnosis (magnification $\times 1000)$. At least 15 crypts were evaluated per subject and per method in sections from one 
or two, and rarely three different biopsies. A crypt was analysed if the entire length of the crypt was visible in the longitudinal section and the base of the crypt contacted the muscularis mucosa.

In each crypt column - that is the single column of epithelial cells lining one side of a sectioned crypt meeting the criteria - the total number of cells and the number of labelled cells and their position in the crypt column relative to the base of the crypt were noted. In the BrdU assay, a cell was considered positive if there was any nuclear staining present. For the PCNA assay faint staining was seen frequently, probably reflecting the relatively long half life of immunoreactive PCNA $^{19}$ and the fact that PCNA is expressed in phases of the cell cycle other than $\mathrm{S}$ phase. For comparison with BrdU, therefore, only the strongly stained nuclei, which are assumed to be in S phase, were counted.

The total number of cells, the number of labelled cells, and the proliferation index, which is the ratio of labelled cells to the total number of cells, were determined for each crypt column. The proliferation index was also calculated separately for each of five compartments of equal size into which each crypt column was divided. The same procedure was repeated for percentiles of the crypt column, and a running mean over five percentiles was calculated to smooth the distribution.

To estimate intraobserver variation, four randomly selected cases were counted on two separate occasions by the same observer.

\section{STATISTICAL ANALYSIS}

Non-parametric statistical methods were used whenever possible, because neither the basic data nor the log transformed data were normally distributed. Correlation coefficients were calculated as Spearman rank correlations and comparison between the two methods by the sign test.

Variance component analysis was used to estimate the number of crypts and the number of biopsy samples that must be evaluated for each method to classify an individual correctly. A nested variance component model with random effects was calculated with the patient as unit and crypt and biopsy specimens as classification

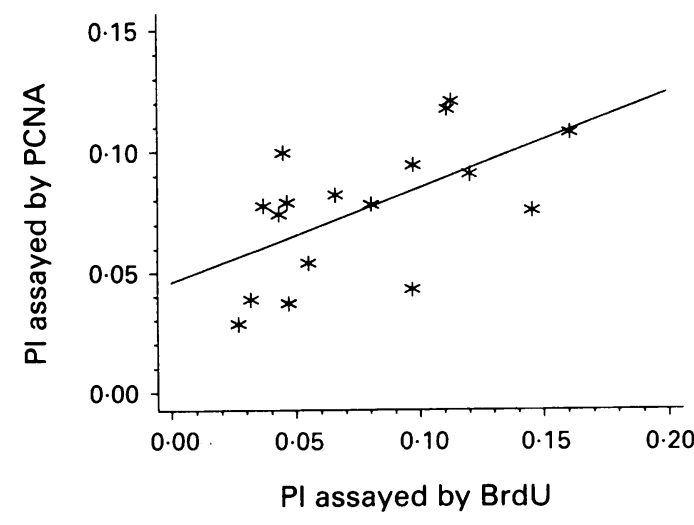

Figure 1: Correlation of individual proliferation indices $(P I)$ measured by the BrdU and by the PCNA method. $\left(r_{\mathrm{s}}=0 \cdot 6\right.$, $p<0.05 ; b=0.39$.) variable. ${ }^{20}$ The coefficient of variation (SD $(100 \%) /$ mean) was calculated from this variance component model for each variable of the model. The variance within a patient was calculated by:

$$
\mathrm{S}_{\mathrm{p}}{ }^{2}=\frac{\mathrm{S}_{\mathrm{c}}{ }^{2}+\mathrm{n} \mathrm{S}_{\mathrm{b}}{ }^{2}}{\mathrm{nb}}
$$

where: $S_{p}{ }^{2}=$ variance of the sample mean per determinant; $S_{c}{ }^{2}=$ variance of crypts (from the variance component analysis); $\mathrm{S}_{\mathrm{b}}{ }^{2}=$ variance of biopsies (from the variance component analysis); $\mathrm{n}=$ number of crypts; $\mathrm{b}=$ number of biopsy specimens. The $95 \%$ confidence intervals $(\mathrm{CI})$ was estimated by: $95 \% \mathrm{CI}=1.96{\sqrt{\mathrm{S}_{\mathrm{p}}}}^{2}$ (see reference 20).

\section{Results}

\section{PROLIFERATION INDEX}

About 37000 colonic epithelial cells were evaluated per method and more than 15 crypts were evaluated per subject. When scoring only the strongly reactive nuclei in the PCNA assay, which were assumed to represent the $S$ phase cells, the individual proliferation indices between the two methods showed a Spearman correlation coefficient of $r_{s}=0.6(p=0.011)$ (Fig 1). The regression equation was estimated as PCNA $=0.0459+0.3912 \mathrm{BrdU}(\mathrm{p}=0.015)$. The mean proliferation index in the study group obtained by the BrdU technique $(7 \cdot 79 \%)$ was virtually equivalent to that obtained by the PCNA technique $(7 \cdot 64 \%)(p=0 \cdot 8)$. Inclusion of the minor reactivity in the PCNA assay gave a mean proliferation index of $9 \cdot 1 \%$ and the correlation failed to be significant. A statistically significant difference between the two methods was found in the total number of cells per crypt column, the crypts appearing longer in the PCNA assay (Table I). The mean proliferation indices within compartments were nearly identical for both assays. Slightly higher proliferation indices were found for the 4th and 5th compartments with the BrdU than with the PCNA technique.

Figure 2 shows the percentile proliferation indices obtained with the two methods. The proliferation index peaked at the 23 rd percentile with the PCNA method. BrdU showed two peaks at the 18th and the 24th percentile. The proliferation index was slightly lower with $\mathrm{BrdU}$

TABLE I Epithelial cell proliferation assayed by proliferation cell nuclear antigen (PCNA) and 5-bromo-2-deoxyuridine $(B r d U)$ in 17 patients with no gastrointestinal disorder

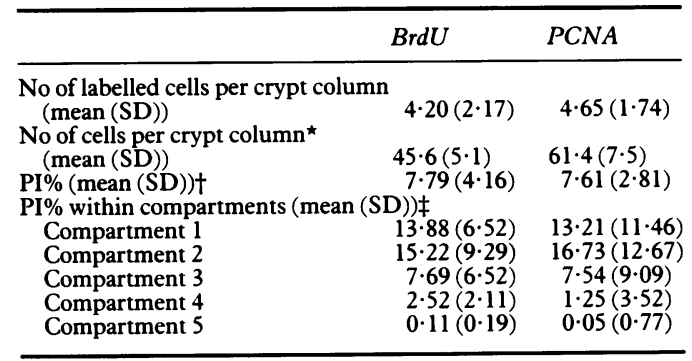

«Difference statistically significant, two sided $\mathrm{p}$ value: $<0 \cdot 05$; $+\mathrm{PI} \%$, proliferation index in per cent; percentage of labelled cells calculated for each patient separately; fpercentage of labelled cells calculated for each compartment and each patient separately. 


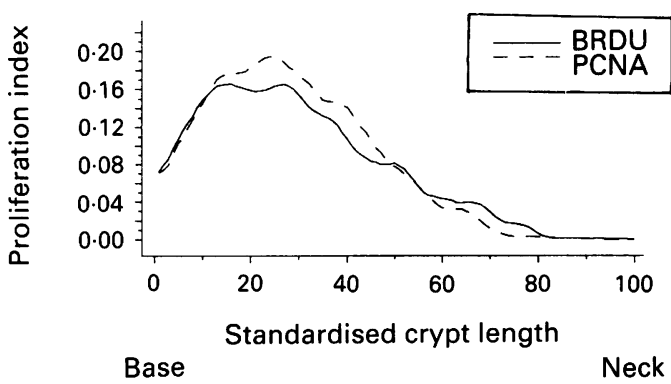

Figure 2: Distribution of the proliferation index in colonic crypts of 17 patients with no gastrointestinal disorder assayed by $B r d U$ and PCNA.

than with PCNA between the 20th and 42nd percentiles and higher between the 60th and the 80th percentile.

\section{INTRAOBSERVER VARIATION}

Re-evaluation of four randomly selected subjects showed a $\mathbf{9 4 . 9 \%}$ agreement on proliferation index in the case of BrdU and a $90.3 \%$ agreement in the case of PCNA. The percentage value of agreement on the total number of cells per crypt column, was $99 \cdot 1 \%$ for BrdU and $97 \cdot 3 \%$ for PCNA.

\section{INDIVIDUAL VARIATION}

Variance component analysis was used to calculate the contribution of the variations within subjects, biopsy samples, and crypts to the total variation of the proliferation index per method. The variance within subjects and within biopsy samples was found to be higher for the BrdU method than the PCNA method, but the variance within crypts was equal (Table II).

Variance component analysis was also used to calculate the required number of crypts (equation [1] resolved for $\mathbf{n}$ ) to estimate the proliferation index for a subject within a $95 \%$ confidence interval with one, two, or three biopsy samples separately for each method (Table III). The gain in precision by taking two biopsy samples compared with one is striking for both methods; this effect is because of variation between biopsy specimens within subjects. The difference

TABLE II Variation coefficients for subjects, biopsy samples, and crypts calculated separately for the PCNA and the BrdU assay in a variance component analysis

\begin{tabular}{llll}
\hline & Subjects & $\begin{array}{l}\text { Biopsy } \\
\text { samples }\end{array}$ & Crypts \\
\hline Coefficient of variation, PCNA & $33 \cdot 0 \%$ & $14 \cdot 7 \%$ & $40 \cdot 0 \%$ \\
Coefficient of variation, BrdU & $49 \cdot 0 \%$ & $16 \cdot 3 \%$ & $40 \cdot 0 \%$ \\
\hline
\end{tabular}

Abbreviations the same as in Table I.

TABLE III No of crypts required per biopsy sample to assess cell proliferation and to estimate an individual proliferation index within defined $95 \%$ confidence intervals

\begin{tabular}{|c|c|c|c|c|c|c|c|c|c|}
\hline \multirow[b]{2}{*}{ No of biopsy samples } & \multicolumn{3}{|c|}{$P I \pm 2 \cdot 0 \%$} & \multicolumn{3}{|c|}{$P I \pm 2 \cdot 5 \%$} & \multicolumn{3}{|c|}{$P I \pm 3.0 \%$} \\
\hline & 1 & 2 & 3 & 1 & 2 & 3 & 1 & 2 & 3 \\
\hline $\begin{array}{l}\text { PCNA } \\
\text { BrdU }\end{array}$ & $\infty$ & $\begin{array}{l}13(26)^{\star} \\
23(46)\end{array}$ & $\begin{array}{l}6(18) \\
7(21)\end{array}$ & $\begin{array}{l}30(30) \\
\infty\end{array}$ & $\begin{array}{l}5(10) \\
7(14)\end{array}$ & $\begin{array}{l}3(9) \\
4(12)\end{array}$ & $\begin{array}{l}10(10) \\
15(15)\end{array}$ & $\begin{array}{l}3(6) \\
4(8)\end{array}$ & $\begin{array}{l}2(6) \\
2(6)\end{array}$ \\
\hline
\end{tabular}

${ }^{\star}$ In parenthesis: total no of crypts for evaluation. Abbreviations the same as in Table I.
TABLE IV Effects of various fixatives on the comparative intensity of immunostaining assayed by PCNA and BrdU in two colonic biopsy specimens from each of three patients

\begin{tabular}{lll}
\hline & \multicolumn{2}{l}{ Immunoreactivity } \\
\cline { 2 - 3 } Fixation & PCNA $($ 19A2) & BrdU \\
\hline Buffered formalin 10\% & - & - \\
Ethanol 70\% & + & ++ \\
Methanol 70\% & + & ++ \\
Methanol 99\% & +++ & +++ \\
'Primafix't & ++ & +++ \\
\hline
\end{tabular}

Scored as: negative $(-)$, weakly positive $(+)$, positive $(++)$, strongly positive $(+++){ }^{\star}$ fixation time for $18-24 \mathrm{~h}$ at $4^{\circ} \mathrm{C}$; talcoholic fixative (Camon, Wiesbaden, Germany).

Abbreviations as in Table I.

between the two methods is most obvious, when the precision of the point estimate is allowed to include a $95 \%$ confidence interval of $\mathrm{PI} \pm 2 \cdot 5 \%$. Such a precision cannot be achieved by using one biopsy sample with the BrdU method, whereas PCNA can provide a point estimate within PI士 $2.5 \%$ by using one biopsy sample with 30 evaluated crypts. Estimation of a subject's proliferation index with the same precision using two biopsy samples requires 10 crypts in total for PCNA and 14 for BrdU. Evaluating more than two biopsy samples leads to an alignment in the number of crypts required to achieve a certain precision.

\section{METHODS}

Differences in time and type of fixation affect the intracellular staining pattern - that is, the strength of the staining and therefore the number of labelled cells. ${ }^{2122}$ Various fixatives were tested (Table IV). The best results were obtained using both methods with absolute methanol fixation at $+4^{\circ} \mathrm{C}$ for $18-24$ hours. Staining intensity in the PCNA assay also introduced the Saponin digestion step. Staining was optimal after 18-24 hours of fixation, but less intense after more than 48 hours of fixation. This effect was no longer seen when the DNA denaturation step in the PCNA protocol was replaced by the Saponin digestion step. Saponin pretreatment also resulted in an augmentation of PCNA staining and a decrease in background staining.

\section{Discussion}

In this study on biopsy samples from colonic mucosa of patients with no colonic disorders, the comparison between two methods of assessing cell proliferation showed a significant correlation $\left(r_{s}=0.6, p<0.05\right)$. Mean proliferation index $(7 \cdot 79,7 \cdot 64$ respectively) as well as its distribution within crypts was similar with the two methods in the study group.

The BrdU index obtained in this material was higher than that reported by Risio et $a l,{ }^{23}$ and lower than that reported in other studies. ${ }^{24}$ Different laboratory practices do not permit direct comparison of values; for example, Wilson et $a l^{24}$ used hyperbaric gas phase during incubation but a lower BrdU concentration in the medium, Risio $e ~_{a l}{ }^{23}$ did not add fluorodeoxyuridine to the medium to enhance BrdU uptake. Our results agree in the distribution of prolifera- 
tion indices by compartments, in that mostly the highest proliferative activity was found in the second compartment when five compartments were calculated. ${ }^{25}$

In this comparative study PCNA data resembled BrdU data when scoring was restricted to the more strongly reactive PCNA nuclei. Inclusion of all reactive nuclei gave PCNA values, which were on average about $20 \%$ higher than BrdU labelling. This variability in strength of nuclear reactivity has also been briefly reported by Battersby et al. ${ }^{16}$ On the other hand, Galand $e t a l^{15}$ showed in their comparison with $\left[{ }^{3} \mathrm{H}\right]$-thymidine labelling that $19 \mathrm{~A} 2$ resulted in $\mathrm{S}$ phase cells becoming stained, when methanol fixation was used. But, they did not report a great variability in the intensity of nuclear staining.

The variance component analysis shows that the variation between biopsy specimens of one subject is larger for the BrdU method compared with the PCNA method. Basically, the intraindividual variability can be caused either by real variability from site to site within the circle of the rectosigmoidal junction or by variability in the sample preparation. The higher variability between biopsy samples in the BrdU method is likely to be because of the second reason - that is, this method is more affected by the conditions of incubation and subsequent processing. Therefore, in terms of precision for a given combination of crypts and biopsy specimens from a subject, the PCNA method seems preferable. Because of the variability between biopsy samples, the gain in precision of defining a subject's proliferation index by taking two biopsy samples compared with one is remarkable for both methods. This finding implies that not only is the total number of evaluated crypts a crucial criterion for a precise estimation of a proliferation index, but also the number of biopsy samples per subject being used for the evaluation. It seems worthwhile to include at least two biopsy samples in the evaluation, for the additional gain in precision is the highest when increasing the number of biopsy samples from one to two samples.

Type of fixation may influence the accessibility of the PCNA protein to antibody detection" is although results from other studies do not support this conclusion. ${ }^{14}{ }^{16}$ The use of absolute methanol in the PCNA protocol for fixation of the tissue yielded the most consistent staining results in our study. This finding is in line with the hypothesis that with this fixation the insoluble form of PCNA, which is associated with the DNA replication sites, can be detected." Galand $e t a l^{15}$ also found that PCNA shows best agreement with $\left[{ }^{3} \mathrm{H}\right]$-thymidine labelling when using methanol fixation. Our finding concerning dependency of staining intensity on duration of fixation has also been made by Battersby et al. ${ }^{16}$ The introduction of Saponin in the PCNA protocol before the application of the PCNA antibody was helpful in that PCNA could be identified even after more than two weeks of fixation. This step prolonged the localisation of PCNA immunoreactivity in time, which is especially useful in multicentre studies, where biopsy specimens have to be sent from several endoscopy units to a central laboratory.
The higher total number of cells per crypt column in the PCNA assay compared with the BrdU assay was suspected to be as a result of an observer effect during the counting process. The slightly stronger staining of labelled cells in the case of BrdU compared with PCNA may facilitate the identification of labelled nuclei. The faster counting could then lead to fewer actually seen nuclei in the BrdU assay compared with PCNA. Re-examination of four randomly selected patients for both methods showed a high agreement on the average total number of cells for each subject. Thus, the observer effect is not a plausible explanation of the phenomenon. A difference because of the handling of the specimens can also be excluded, because specimens for both BrdU and PCNA assay were equally flattened on a filter paper before fixation. An increased cell loss during incubation with BrdU as another possible explanation is not supported by published works. ${ }^{26}$ The significant difference in the number of cells per crypt column needs further investigation with regard to its cause and its possible confusing effects on the analysis of labelled cell distribution.

It is concluded that assuming nuclear non-S phase staining is faint comparative to staining of replicating cells, PCNA antibodies may be reliable markers to detect in situ cells with $S$ phase contents. To detect $S$ phase cells by means of PCNA assay, scoring procedures have to be strictly standardised to consistently score only strongly stained nuclei, which may be achieved best by using image analysis. If these conditions are not met, we assume that it might be more reliable to regard PCNA as a marker with its own evidence to characterise proliferative activity by scoring all PCNA reactive nuclei as positive regardless of staining intensity.

The authors wish to thank Mrs E Jumpertz for her excellent technical assistance.

1 Lippman SM, Lee JS, Lotan R, Hittelman W, Wargovich MJ, Hong WK. Biomarkers as intermediate end points in chemopreventive trials. F Natl Cancer Inst 1990; 82: 555-60. 2 Williamson RCN, Malt RA. Promotion of intestinal carcinogenesis by adaptive mucosal hyperplasia. In: Appleton DR Sunter JP, Watson AJ, ed. Cell proliferation in the gastrointestinal tract. London: Pitman, 1980: 303-15.

3 Lipkin M. Early identification of population groups at high risk for gastrointestinal cancer. In: Malt RA, Williamson RCN, ed. Colonic carcinogenesis. Lancaster: MTP Press, 1981: 31-46.

4 Wargovich MJ, Medline A, Bruce WB. Early histopathologic events to evolution of colon cancer in $\mathrm{C} 57 \mathrm{BL} / 6$ and $\mathrm{CF} 1$ mice treated with 1,2-dimethylhydrazine. F Natl Cancer Inst 1983; 71: 125-31.

5 Preston-Martin S, Pike MC, Ross RK, Jones PA, Henderson BE. Increased cell division as a cause of human cancer. Cancer Res 1990; 50: 7415-21.

6 Lipkin M, Blattner WE, Fraumeni JF, Lynch HT, Deschne $\mathrm{E}$, Winawer S. Tritiated thymidine labelling distribution as a marker for hereditary predisposition to colon cancer. Cancer Res 1983; 43: 1899-904

7 Gratzner HG. Monoclonal antibody to 5-bromo and 5-iododeoxyuridine: A new reagent for detection of DNA replication. Science 1982; 218: 474-6.

8 Gerdes J, Lemke H, Baisch H, Wacker HH, Schwab U, Stein H. Cell cycle analysis of a cell proliferation-associated human nuclear antigen defined by the monoclonal antibody Ki-67. F Immunol 1984; 133: 1710-5.

9 Ogata K, Kurki P, Celis JF, Nakamura PM, Tan FM Monoclonal antibodies to a nuclear protein (PCNA cyclin) associated with DNA replication. Exp Cell Res 1987; 168

0 Morris GF, Mathews MB. Regulation of proliferating cell nuclear antigen during the cell cycle. F Biol Chem 1989; 264 13856-64. 
11 Bravo R, Macdonald-Bravo $H$. Existence of two populations of cyclin proliferating cell nuclear antigen during the cell cycle: association with DNA replication sites. $\mathcal{F}$ Cell Biol 1987; 105: 1549-54

12 Coltrera MD, Gown AM. PCNA/Cyclin expression and BrdU uptake define different subpopulations in different cell lines. uptake define different subpopulations

13 Yamada K, Yoshitake K, Sato M, Ahnen DJ. Proliferating cell nuclear antigen expression of the rat. Gastrenterology 1992; 103: $160-7$

14 Garcia RL, Coltrera MD, Gown AM. Analysis of proliferative grade using anti-PCNA/Cyclin monoclonal antibodies fixed, embedded tissues. Am $\mathcal{Y}$ Pathol 1989; 134: 733-9.

15 Galand P, Degraef C. Cyclin/PCNA immunostaining as an alternative to tritiated thymidine pulse labelling for marking $S$ phase cells in paraffin sections from animal and human tissues. Cell Tissue Kinet 1989; 22: 383-92.

16 Battersby S, Anderson TJ. Correlation of proliferative activity in breast tissue using PCNA/Cyclin. Hum Pathol 1990; 21 781 .

17 Fireman Z, Rozen P, Fine N, Chetrit A. Reproducibility studies and effects of bowel preparations on measurements studies and effects of bowel preparations on measurements

18 Shamsuddin AKM, Weiss L, Phelps PC, Trump BF. Changes in human colon mucosa adjacent to and remote from 9 Hall PA, Levison DA, Woods AL, et al. Proliferating cel nuclear antigen (PCNA) immunolocalization in paraffin sections: an index of cell proliferation with evidence of deregulated expression in some neoplasms. F Pathol 1990; 162: 285-94.

20 Snedecor GW, Cochram WG. Statistical methods. Ames, Iowa: Iowa State University Press, 1967: 287

21 Bravo R, Macdonald-Bravo H. Changes in the nuclear distribution of cyclin (PCNA) but not its synthesis depend on DNA replication. EMBO J 1985; 4:655.

22 Kurki P, Ogata K, Tan EM. Monoclonal antibodies to proliferating cell nuclear antigen (PCNA)/cyclin as probes orescence microscop and flow cytometry. F Immunol Methods 1988; 109: 49-59.

23 Risio M, Coverlizza S, Ferrari A, Candelaresi GL, Rossini FP. Immunocytochemical study of epithelial cell proliferation in hyperplastic polyps, adenomas, and adenocarcinomas of the large bowel. Gastroenterology 1988; 94: 899-906.

24 Wilson RG, Smith AN, Bird CC. Immunohistochemical detection of abnormal cell proliferation in colonic mucosa of subjects with polyps. F Clin Pathol 1990; 43: 744-7.

25 Risio M, Lipkin M, Candelaresi GL, Bertone A, Coverlizza S Rossini FP. Correlations between rectal mucosa cell proRossini FP. Correlations between rectal mucosa cell pro-

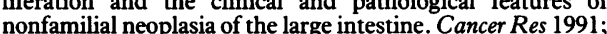
51: 1917-21.

26 Eastwood GL, Trier JS. Organ culture of human recta mucosa. Gastroenterology 1973; 64: 375-82. 